\title{
A Multi-Scale Investigation of Global Electrical Heterogeneity: Effects of Body Habitus, Respiration, and Tissue Conductivity
}

\author{
Erick A Perez-Alday ${ }^{1}$, Haibo $\mathrm{Ni}^{2}$, Christopher Hamilton ${ }^{1}$, Annabel Li-Pershing ${ }^{1}$, Bernard Jaar ${ }^{3}$, Jose \\ M Monroy-Trujillo ${ }^{3}$, Michelle Estrella ${ }^{3,4}$, Rulan Parekh ${ }^{3,5}$, Henggui Zhang ${ }^{6}$, Larisa Tereshchenko ${ }^{1,3}$ \\ ${ }^{1}$ Oregon Health \& Science University, Portland, OR, USA; ${ }^{2}$ University of California Davis, Davis, \\ CA, USA; ${ }^{3}$ Johns Hopkins University, Baltimore, MD, USA; University of California San Francisco, \\ San Francisco, CA, USA; ${ }^{5}$ University of Toronto, Toronto, Canada; ${ }^{6}$ University of Manchester, \\ Manchester, United Kingdom.
}

\begin{abstract}
Extracardiac factors such as respiration, fluid overload and body habitus have important effects on the ECG voltage. Vectorcardiographic (VCG) Global Electrical Heterogeneity $(G E H)$ is associated with sudden cardiac death (SCD). Risk of SCD is especially high in end-stage renal disease patients (ESRD) on dialysis. However, extracardiac factors challenge ECG interpretation in ESRD patients. The effects of extracardiac factors on GEH have not been fully studied. To assess effects of extracardiac factors on ECG, we conducted a multi-scale study. An experimental data of ESRD patients and a previously developed biophysically detailed heart-torso model were used to investigate the effects of respiration, fluid overload and body habitus on the VCG and GEH.
\end{abstract}

\section{Introduction}

Vectorcardiographic (VCG) Global Electrical Heterogeneity (GEH) parameters are associated with sudden cardiac death (SCD) [1]. Both intracardiac and extracardiac factors can affect the ECG voltage, which can challenge the clinical interpretation of GEH. For example, both low [2] and high [3] sum absolute QRST integral (SAI QRST) was associated with increased risk of ventricular arrhythmia. In addition, interpretation of ECG voltage is especially difficult in patients with end-stage renal disease (ESRD) on dialysis, who frequently experience fluid overload, and have increased risk of cardiovascular disease (CVD) and heart failure, leading to pulmonary congestion, manifested by low ECG voltage. Fluid loss, on the other hand, can cause a decrease of body conductivity which usually results in increased amplitude of the ECG voltage [4], which can mimic ECG-left ventricular hypertrophy[5].

Clinically it is important to differentiate whether ECG voltage and GEH changes are due to cardiac, or extracardiac factors. The effects of several extracardiac factors on ECG voltage and body surface potential (BSP) were studied previously [6, 7]. In this studies, either experimental models or in silico approaches were employed to assess the dependence of ECG voltage and BSP on the tissue conductivity and body habitus $[5,7,8]$. However, fewer studies have combined both experimental and modelling data to focus on VCG or GEH parameter.

The present multi-scale (in silico and clinical observational cohort) study aims to investigate the effect of respiration, tissue conductivity and heart position (body habitus) on vector magnitude (VM) and GEH in ESRD patients on dialysis.

\section{Methods}

\subsection{Experimental data}

The prospective cohort Predictors of Arrhythmic and Cardiovascular risk in End-stage renal disease (PACE) data $[4,9]$ was analysed. The study was approved by the Institutional Review Board, and all study participants signed written consent form before enrolment.

PACE participants underwent 5 minutes $(1000 \mathrm{~Hz}) \mathrm{XYZ}$ Frank ECG recording using Norav 1200 M PC ECG machine (Norav Medical Ltd, Thornhill, ON, Canada). Only sinus rhythm data was included in this study. From the 5 minutes ECG, two cardiac cycles recorded at different respiration phases during normal breathing - one at maximum inspiration and another at maximum expiration - were extracted. Each beat was inspected to ensure there was low noise and no distortion. Amplitudes of QRS complex and T-wave were measured on the VM. GEH parameters (spatial QRS-T angle, spatial ventricular gradient (SVG) magnitude (mag), azimuth (azi), and elevation (elev), and SAI QRST) were calculated for each beat, as previously described. GEH software code and equations are provided at:

https://physionet.org/physiotools/geh./ 


\subsection{Modelling data}

A previously developed three dimensional (3D) biophysically detailed computational model of the human ventricles (Figure 1-Ai) was used to simulated normal ventricular activation [10]. The ventricular model is segmented into the major distinctive electrically heterogeneous regions (Figure 1-Aii). The models incorporated anatomical structures and detailed electrophysiological heterogeneity with cellular electrophysiology being previously described (Figure 1Aiii) [10].

The ventricular model was incorporated in a previously developed heart-torso model [11], which considers the presence of blood masses, lung, liver, stomach, kidneys, spinal cord, ribs and fat tissue (Figure 1-B), each with different electrical conductivity based on previously published data (Table 1) $[6,12,13]$. Boundary element method was used to compute the BSP resulting from the simulated current density obtained from the electrical activity of the ventricular tissue-model. More details of the models have been previously described [10].

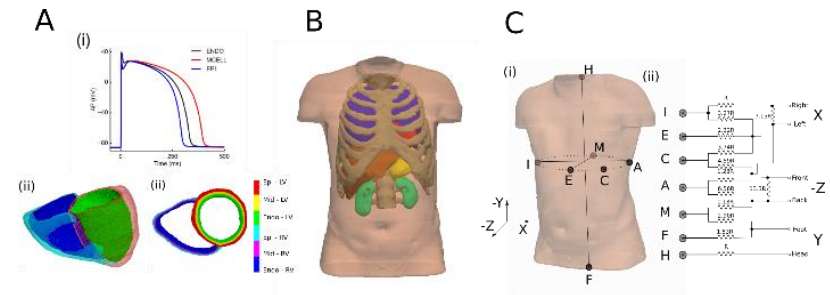

Figure 1. Schematic illustration of the modelling procedure. A) Ventricular model with the anatomical structures and electrophysiological heterogeneity. B) Torso model with heterogeneous conductivity considered. C) Frank XYZ ECG system and equivalent circuit.

\subsection{Modelling Frank ECG system}

To simulate the original XYZ Frank ECG system, the element of the thorax mesh closer to the position of the electrodes from the Frank system was selected (Figure 1Ci) [14]. Then the circuit equations were solved to obtain the $\mathrm{X}, \mathrm{Y}$ and $\mathrm{Z}$ leads from the simulated electrodes (Figure 1-Cii). Amplitudes of QRS complex and T-wave were measured on the VM. Finally, GEH parameters were calculated for each simulated case.

\subsection{Modelling tissue conductivity}

The tissue conductivities used in this study are based on experimental data and previous studies $[6,12,13]$. The conductivity values taken as normal were Lungs $0.08 \mathrm{~S} / \mathrm{m}$, Blood masses $0.60 \mathrm{~S} / \mathrm{m}$, Fat tissue $0.05 \mathrm{~S} / \mathrm{m}$, Bones 0.005 $\mathrm{S} / \mathrm{m}$, Kidneys $0.07 \mathrm{~S} / \mathrm{m}$, Liver $0.15 \mathrm{~S} / \mathrm{m}$, Stomach 0.12
$\mathrm{S} / \mathrm{m}$, averaged thorax $0.22 \mathrm{~S} / \mathrm{m}$. To simulate fluid in the interstitium, a range of conductivity parameters of the averaged torso was considered in two models: first in a homogenous torso, and then in the model that considered the rest of the tissues (i.e., heterogeneous torso). Results from both cases were compared.

\subsection{Modelling respiration}

To simulate respiration, a range of conductivity parameters of the lungs was considered in two models. First, only the lungs were placed within a homogenous torso. Then, the remaining tissues were incorporated (heterogeneous torso) and only the conductivity of the lungs was varied in both cases. For the comparison against experimental data, the conductivity of the lungs in the simulated data was set to 0.03 and $0.13 \mathrm{~S} / \mathrm{m}$ for inspiration and expiration, respectively.

\subsection{Modelling body habitus}

To simulate different body habitus, we considered two settings, asthenic and hypersthenic body types, which we compared against normosthenic body habitus. For the asthenic body habitus, we rotated the long axis of the heart of the heart $+45^{\circ}$ on the Z-axis compared to normosthenic. To simulate hypersthenic body type, we rotated the long axis of the heart $-45^{0}$ on the $\mathrm{Z}$-axis compared to the normosthenic position. All the tissues inside the body were modified in each case to fit the position of the heart and avoid tissue overlapping. In these simulations, the size or conductivity of the tissues were kept constant.

\section{Results}

\subsection{Experimental data}

From the PACE participants, 254 subjects (mean age $54.6 \pm 13.5 y$; $56 \%$ male; $79 \%$ African American) were included in this study. The subjects included had a history of hypertension (99\%), diabetes (54\%), and cardiovascular disease $(44 \%)$. They have an average body mass index (BMI) of 30.2 \pm 8.1 . The average left ventricular ejection fraction (LVEF) was $66.7 \pm 11.5 \%$.

\subsection{Modelling results}

\subsubsection{Effects of tissue conductivity}

When the conductivity was varied in the homogenous torso, a decrease in the QRS and T-wave VM amplitudes was observed consistently with an increase in the conductivity (e.g. resulting from fluid overload, oedema, and pulmonary congestion). In the case of the homogenous 
torso, the changes of the QRS and T-wave amplitudes were the same. For the heterogeneous torso, these changes were smaller for the T-wave compared to the QRS magnitude (Figure 2).

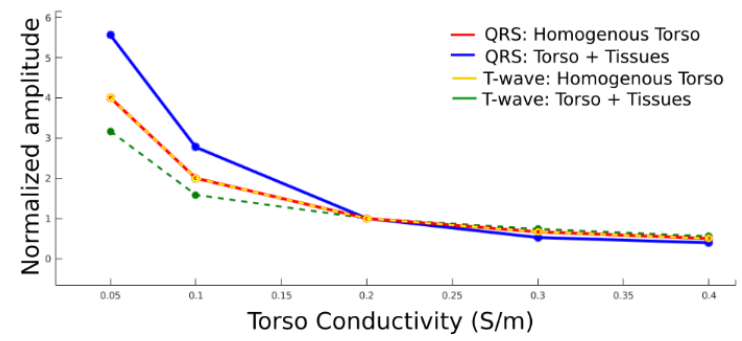

Figure 2. Plot of the QRS VM (solid) and T-wave VM (dashes) normalized amplitude vs Conductivity $(\mathrm{S} / \mathrm{m})$ of the homogenous torso (red/yellow) and torso with the tissue inhomogeneity (blue/green).

\subsubsection{Effects of respiration}

Changes in the lungs conductivity produced a change in the VCG amplitude. Observed changes for the QRS and Twave amplitudes were dramatically different. When the lungs conductivity was decreased (peak of inspiration), the QRS VM amplitude decreased in both homogenous and heterogeneous torso. On the contrary, the decrease in lung conductivity (peak of inspiration) resulted in an increase in the T-wave VM amplitude in the homogenous torso model. In contrast, a U-shaped relation between a lung conductivity and a T-wave VM amplitude was observed for the heterogeneous torso (Figure 3).



Figure 3. Plot of the QRS (solid) and T-wave (dashed) VM normalized amplitude vs Conductivity $(\mathrm{S} / \mathrm{m})$ of the lungs within a homogenous torso (red/yellow) and within torso with the tissue inhomogeneity (blue/green).

\subsubsection{Effects of body habitus}

The position of the heart had an important effect on the amplitude and angles of the VCG. The large changes were observed in the QRS-T angle and the SVG azimuth (Figure 4). The difference of rotation from $-45^{0}$ to $+45^{0}$ of the heart axis along the Z-axis (Figure 4-i) yield to a difference of QRST angle from $22^{\circ}$ for asthenic body habitus (Figure Aii) to $151^{\circ}$ for hypersthenic body habitus (Figure $4 \mathrm{C}$-ii).
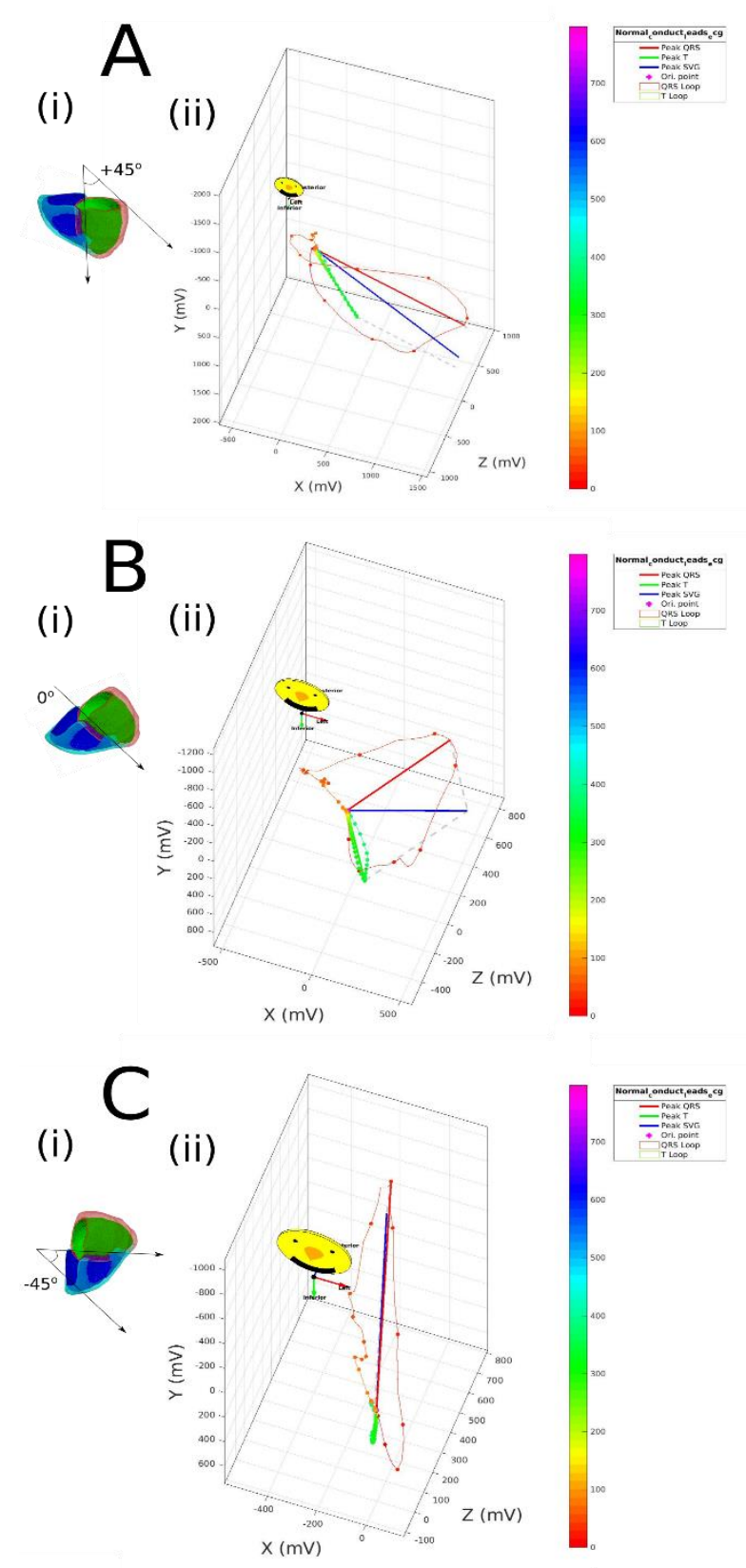

Figure 4. Plot of the VCG loops of the different body habitus simulations: A) Asthenic $\left(+45^{0}\right.$ rotation $)$ B) Normo-sthenic $\left(0^{0}\right.$ rotation $)$; and C) Hypersthenic $\left(-45^{0}\right.$ rotation).

\subsection{Modelling vs experimental GEH parameters}

Table 1 shows that the respiration effect on GEH observed in ESRD patients and in the simulated data, was generally consistent. However, modeling over-estimated the effect of respiration on GEH, which was especially prominent for effect on SVG magnitude (6\% difference). 
Our simulations showed that a body habitus produced the largest effect on GEH. Change in the conductivity of interstitium produced the largest effect on ECG amplitudes. Respiration produced very small changes (Table 2), as compared to conductivity and body habitus.

Table 1. GEH parameters during inspiration (Insp) and expiration (Exp), comparison between experimental and simulated data.

\begin{tabular}{lcccc}
\hline & \multicolumn{2}{c}{ Modelling } & \multicolumn{2}{c}{ Experimental } \\
\cline { 2 - 5 } & Insp. & Exp. & Insp.(SD) & Exp.(SD) \\
\hline SAI, & 203.9 & 211.8 & 215.2 & 222.8 \\
mV*ms $^{*}$ & & & $(76.3)$ & $(79.8)$ \\
QRST ang, $^{\circ}$ & 125.9 & 127.74 & 113.45 & 116.21 \\
& & & $(34.99)$ & $(36.44)$ \\
Wilson SVG & 67.6 & 74.0 & 69.0 & 72.3 \\
mag, mV & & & $(31.9)$ & $(34.5)$ \\
SVG elev, $^{\circ}$ & 50.9 & 46.16 & 66.5 & 65.6 \\
SVG azi, $^{\circ}$ & 42.7 & 44.3 & 19.4 & 22.9 \\
QRS mag, & 1.3 & 1.4 & 2.47 & 2.63 \\
mV & & & $(1.08)$ & $(1.11)$ \\
T mag, mV & 0.89 & 0.86 & 0.460 & 0.469 \\
& & & $(0.244)$ & $(0.249)$ \\
\hline
\end{tabular}

Table 2. Relative changes on the GEH parameters due to respiration, heart position and tissue conductivity.

\begin{tabular}{|c|c|c|c|c|}
\hline & \multicolumn{2}{|c|}{ Respiration } & \multirow{2}{*}{$\begin{array}{c}\text { Body } \\
\text { type } \\
\text { Simula } \\
\text { ted }\end{array}$} & \multirow{2}{*}{$\begin{array}{c}\text { Thorax } \\
\text { conduct } \\
\text { Simula } \\
\text { ted }\end{array}$} \\
\hline & $\begin{array}{l}\text { Experi } \\
\text { mental }\end{array}$ & $\begin{array}{c}\text { Simula } \\
\text { ted }\end{array}$ & & \\
\hline SAI & $3.5 \%$ & $3.8 \%$ & $50 \%$ & $48 \%$ \\
\hline QRST & $2.4 \%$ & $4.6 \%$ & $81 \%$ & $13 \%$ \\
\hline $\begin{array}{l}\text { Wilson- } \\
\text { SVG mag }\end{array}$ & $5.7 \%$ & $9.4 \%$ & $22 \%$ & $7.3 \%$ \\
\hline SVG elev & $1.3 \%$ & $9.3 \%$ & $42 \%$ & $2.6 \%$ \\
\hline SVG azi & $18 \%$ & $3.7 \%$ & $80 \%$ & $6 \%$ \\
\hline QRS mag & $6.4 \%$ & $7.6 \%$ & $2.9 \%$ & $32 \%$ \\
\hline $\mathrm{T}$ mag & $1.9 \%$ & $3.3 \%$ & $7.7 \%$ & $36 \%$ \\
\hline
\end{tabular}

\section{Discussion and Conclusion}

In this study we observed that the tissue conductivity had non-linear effect on the VM amplitude, in agreement with previous investigations on its effect on ECG voltages $[6,7]$. This nonlinear effect can explain clinically observed dependence of ECG voltage on edema and respiration. Body habitus had an important effect on QRS-T angle and SVG direction. Observed differences in effect of conductivity on QRS and T amplitudes are provocative and warrant further study. Extracardiac factors, such as respiration, edema and body habitus, have main effects in the ECG voltage and direction of the vectors obtained from the VCG loops. Therefore, they must take into account in future ECG studies.

\section{References}

[1] Waks, J.W., C.M. Sitlani, E.Z. Soliman, et al., Global electric heterogeneity risk score for prediction of sudden cardiac death in the general population: the atherosclerosis risk in communities (ARIC) and cardiovascular health (CHS) studies. Circulation, 2016. 133(23): p. 2222-34.

[2] Tereshchenko, L.G., A. Cheng, B.J. Fetics, et al., A new electrocardiogram marker to identify patients at low risk for ventricular tachyarrhythmias: sum magnitude of the absolute QRST integral. Journal of Electrocardiology, 2011. 44(2): $\mathrm{p}$. 208-216.

[3] Tereshchenko, L.G., S. McNitt, L. Han, et al., ECG marker of adverse electrical remodeling post-myocardial infarction predicts outcomes in MADIT II study. PLoS One, 2012. 7(12): p. e51812.

[4] Tereshchenko, L.G., E.D. Kim, A. Oehler, et al., Electrophysiologic substrate and risk of mortality in incident hemodialysis. J Am Soc Nephrol, 2016. 27(11): p. 34133420 .

[5] Abächerli, R., L. Zhou, J.J. Schmid, et al., Correlation relationship assessment between left ventricular hypertrophy voltage criteria and body mass index in 41,806 Swiss conscripts. Annals of Noninvasive Electrocardiology, 2009. 14(4): p. 381-388.

[6] Keller, D.U., F.M. Weber, G. Seemann, et al., Ranking the influence of tissue conductivities on forward-calculated ECGs. IEEE Transactions on Biomedical Engineering, 2010. 57(7): p. 1568-1576.

[7] Rudy, Y., R. Wood, R. Plonsey, et al., The effect of high lung conductivity on electrocardiographic potentials. Results from human subjects undergoing bronchopulmonary lavage. Circulation, 1982. 65(3): p. 440-445.

[8] MacLeod, R.S., Q. Ni, B. Punske, et al., Effects of heart position on the body-surface electrocardiogram. Journal of Electrocardiology, 2000. 33: p. 229-237.

[9] Parekh, R.S., L.A. Meoni, B.G. Jaar, et al., Rationale and design for the predictors of arrhythmias and cardiovascular disease in end stage renal disease (PACE) study. BMC Nephrol, 2015. 16(1): p. 63.

[10]Alday, E.A., H. Ni, C. Zhang, et al., Comparison of electricand magnetic-cardiograms produced by myocardial ischemia in models of the human ventricle and torso. PLoS One, 2016. 11(8): p. e0160999.

[11]Alday, E.A., M.A. Colman, P. Langley, et al., A new algorithm to diagnose atrial ectopic origin from multi lead ECG systems--insights from 3D virtual human atria and torso. PLoS Comput Biol, 2015. 11(1): p. e1004026.

[12]Duck, F.A., Physical properties of tissues: a comprehensive reference book. 2013: Academic Press.

[13]Gabriel, C., A. Peyman, and E. Grant, Electrical conductivity of tissue at frequencies below $1 \mathrm{MHz}$. Physics in Medicine \& Biology, 2009. 54(16): p. 4863.

[14]Frank, E., An accurate, clinically practical system for spatial vectorcardiography. Circulation, 1956. 13(5): p. 737-749.

Address for correspondence:

Erick Andres Perez Alday, PhD,

3181 SW Sam Jackson Park Rd; UHN62; Portland, OR, 97239.

E-mail address: perezald@ohsu.edu 